\begin{tabular}{|l|l|l|l|l|}
\hline Cuadernos de Investigación Geográfica & 2004 & $N^{\circ} 30$ & pp. 169-180 & ISSN 0211-6820 \\
\hline
\end{tabular}

(c) Universidad de La Rioja

\title{
EL GLACIAR ROCOSO DE CERRO KRUND, TIERRA DEL FUEGO, ARGENTINA.
}

\author{
J. Ma . REDONDO VEGA \\ Departamento de Geografía. Universidad de León. Campus de Vegazana s/n. 24071-León. \\ Correo electrónico de contacto: dgejrv@unileon.es
}

\begin{abstract}
RESUMEN: En la Sierra de Alvear, Tierra del Fuego, se ubica el Cerro Krund, que domina el complejo de esquí de Cerro Castor. En la vertiente Sur del Cerro Krund, a $900 \mathrm{~m}$ de altitud, se localiza un glaciar rocoso activo como atestiguan la presencia de hielo intersticial y de grietas en una pista de acceso que lo atraviesa de forma transversal. La zona está sometida a condiciones morfoclimáticas de tipo periglaciar que han sustituido a las glaciares desde la deglaciación iniciada en torno a 10-9 mil años B.P. La dinámica periglaciar y unas favorables condiciones morfoestructurales (fragmentación de la roca, extenso afloramiento al desaparecer el hielo glaciar) y tectónicas (elevada sismicidad de la región) son los factores que han favorecido la génesis del glaciar rocoso.
\end{abstract}

ABSTRACT: In the Sierra Alvear, Tierra del Fuego, the Cerro Krund is located overhead the ski Cerro CastorArea. In the South slope of the Cerro Krund, to $900 \mathrm{~m}$ a.s.l., an active rockglacier is siting as they attest the presence of interstitial ice and of cracks in track slope that crosses it. The area is subjected to morfoclimatic periglacial conditions that have substituted to the glaciers from the initiate deglaciation 10-9 ka. BP aproximately. The periglacial dynamic and some favorable morfo-estructural conditions (fragmentation of the rock, extensive outcrop when the glacier ice has disappeared) and tectonics (regional high seismic character) they are the factors that have favored the rockglacier genesis.

Palabras clave: glaciar rocoso activo, glaciar rocoso de lengua, Cerro Krund, Tierra del Fuego.

Key words: active rockglacier, tongue shaped rockglacier, Cerro Krund, Tierra del Fuego.

Enviado en Septiembre de 2004

Aceptado en Octubre de 2004 


\section{Introducción}

La Sierra de Alvear es un segmento, de dirección este-oeste, de los Andes Fueguinos que constituye la divisoria entre la depresión que ocupa el Lago Fagnano al norte y el valle de Tierra Mayor-Larsifashaj al sur. Una serie de cordales montañosos de dirección norte-sur, delimitados por valles en artesa que desembocan en la depresión Tierra MayorLarsifasaj, constituyen el armazón morfo-estructural de la zona. Entre dos de estos valles de dirección norte-sur, Tristen al este y Las Cotorras al oeste, se eleva de forma enérgica el Cerro Krund (1003m., Fig. 1), en cuya vertiente sur, y aprovechando la morfología de un antiguo circo glaciar, se localiza el glaciar rocoso activo del mismo nombre.

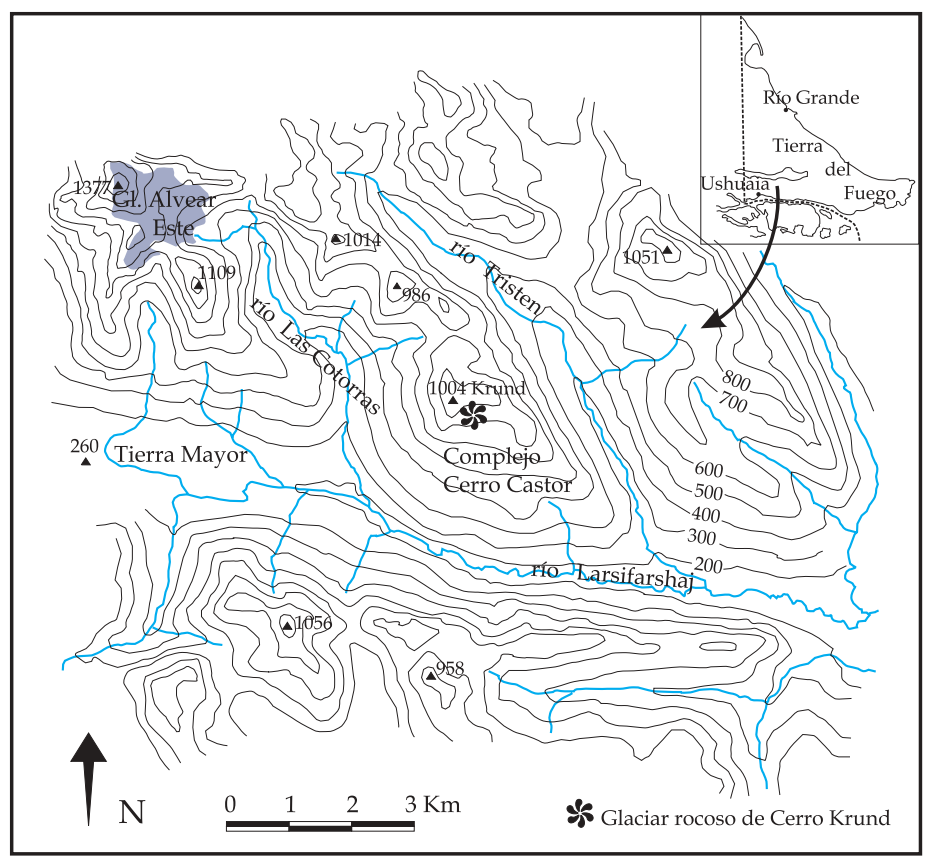

Figura 1. Localización del área de estudio.

Fuente cartográfica: Mapa topográfico de Ushuaia. E. 1/100.000. 2003.

Desde el punto de vista geológico, la vertiente en la que se formó el glaciar del circo arma sobre las series de volcánicas y volcano-clásticas de la formación Lemaire de edad Jurásica. El área se localiza inmediatamente al norte de la falla que sigue el valle Carvajal-Tierra Mayor-Larsifashaj de dirección E-W; la región se caracteriza por el peligro de eventos sísmicos de grado 7 y una frecuencia de 50 años, (Rabassa, dir, 2004).

La zona estuvo ocupada por un glaciar de valle (Coronato, 1995, Coronato y Roig, 1999), entre el Pleistoceno tardío y el comienzo del Holoceno (Rabassa et al., 2000). El 
glaciar estuvo alimentado desde los numerosos circos que ocupan las cabeceras de los cordales montañosos y era tributario del lóbulo principal que se asentaba sobre el Canal Beagle, constituyendo otro eje de primer orden de la glaciación en los Andes Fueguinos (Rabassa et al., 2000). Este glaciar alpino se incorporaba al del Canal Beagle en Bahía Brown, situada a unos $50 \mathrm{~km}$ al E de Ushuaia, aunque mucho antes y siguiendo el actual valle del río Olivia, una difluencia incorporaba una parte de su hielo al lóbulo principal en las inmediaciones de la citada localidad (Coronato y Roig, 1999).

A partir de 10.000 años B.P. se produce la deglaciación persistiendo el hielo sólo en algún pequeño valle y en los circos mejor orientados al S, SE y SW (Coronato 1996). En época histórica se constatan algunos reavances del hielo (PEH) en el ámbito de los circos dejando arcos morrénicos sin colonización vegetal por encima de la línea del bosque (Rabassa et al., 2000).

Por lo que se refiere a las características climáticas de la zona desde el punto de vista fitogeográfico la Sierra de Alvear estaría comprendida dentro de la unidad del bosque caducifolio ( $N$. pumilio) (Tuhkanen, 1992), con temperaturas para el mes más cálido comprendidas entre $9-11^{\circ} \mathrm{C}$, y $-3,5-+2{ }^{\circ} \mathrm{C}$, para el más frío; la duración del periodo vegetativo es algo superior a cuatro meses (está comprendida entre 145-215 días al año), y una precipitación anual de 450-650 mm., (Tuhkanen, 1992). La snow line en las montañas del entorno del Canal de Beagle se establece en torno a los 1000-1100 m (Rabassa et al., 1990).

\section{Caracteres morfológicos}

Si nos situamos al pié del escarpe rocoso de Cerro Krund, se aprecia claramente la morfología en lengua (tongue shaped rock-glacier, Barsch, 1996) del glaciar rocoso. Se ubica sobre el fondo del antiguo circo glaciar, en cuyas paredes posteriores existen extensos afloramientos de vulcanitas fuertemente tectonizadas y afectadas por una intensa crioclastia que se constituyen como su área fuente. El eje principal de la lengua está orientado al S.

La lengua consta de dos partes claramente diferenciadas: desde su raíz hasta la pista de acceso, que lo corta transversalmente, se caracteriza por una mayor pendiente $\left(22^{\circ}\right.$ $26^{\circ}$ ) y una disposición más caótica de sus cantos y bloques; sólo en la parte inferior de este tramo, se distinguen dos crestas y un surco truncado por la pista de acceso mencionada. Hacia abajo, la lengua tiene una menor pendiente, $18^{\circ}$, y se aprecian la sucesión clásica de surcos y crestas, (furrows and ridges, "porridge-like" appearance, Barsch, 1996); un total de cinco crestas (flechas negras) y cuatro surcos (flechas blancas) transversales (Foto 1), terminando en un abrupto talud, frente del glaciar rocoso, que se apoya directamente sobre un umbral rocoso transversal de origen glaciar. En total tiene una longitud modesta de unos $240 \mathrm{~m}$ por algo menos de 100 de ancho.

El frente de los glaciares rocosos activos es siempre un talud muy inclinado cuyo valor viene determinado por el ángulo de reposo del material que lo compone y que tiene que ver, por ello, con su granulometría. En el caso del glaciar rocoso de Cerro Krund el 


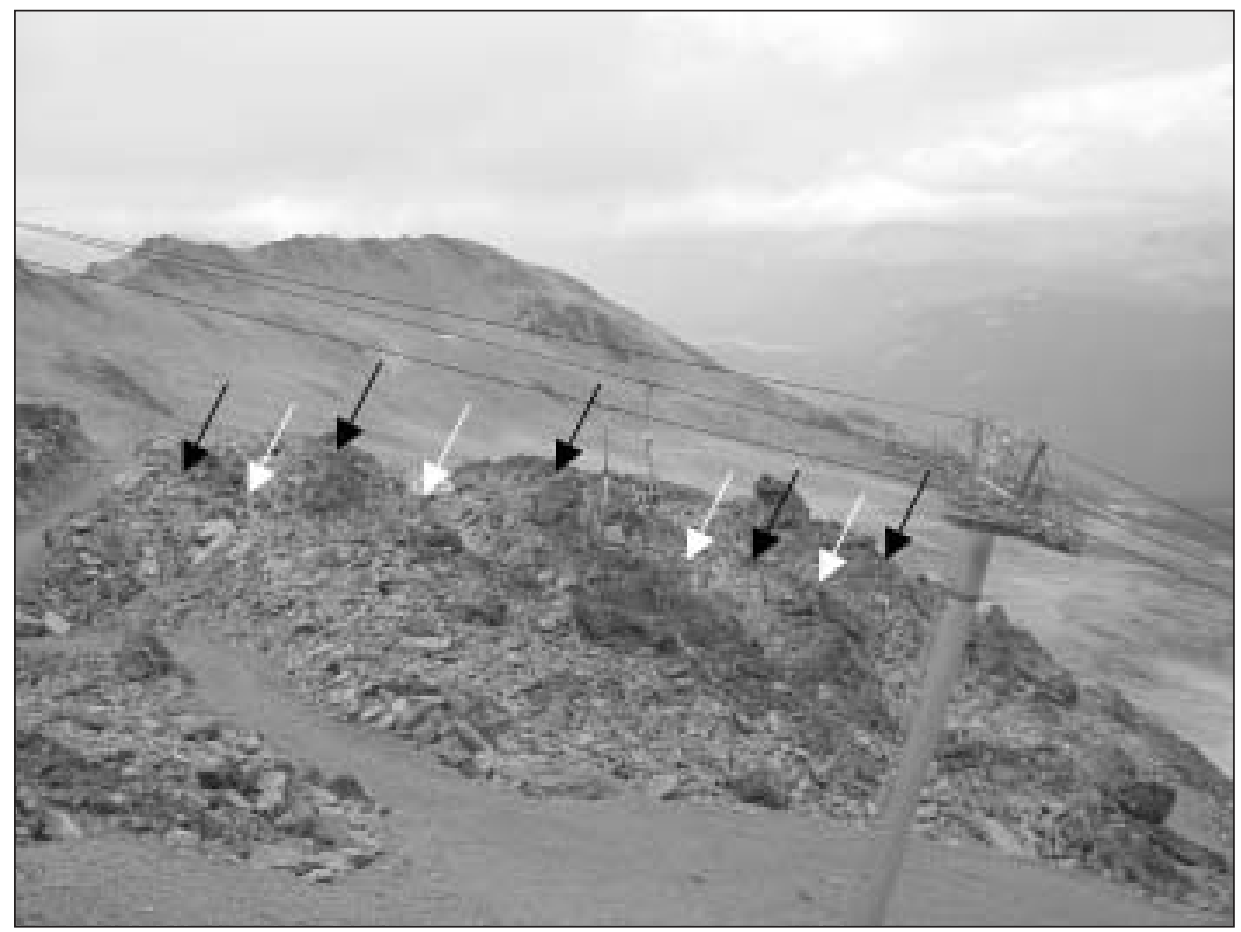

Foto 1. Vista general del sector inferior del glaciar rocoso de Cerro Krund. Son visibles la sucesión de crestas (flechas negras) y surcos (flechas blancas) éstos de tonos más claros debido a la ausencia de colonización liquénica.

frente tiene un ángulo de $34^{\circ}$. Por otro lado, los frentes suelen apoyarse contra, y sobre, cualquier obstáculo (umbral glaciar, depósito morrénico) que hace las funciones de tope contra el cual se desarrolla el glaciar rocoso; en este caso, se trata de un umbral rocoso pulido anteriormente por el paso del hielo ( Foto 2), y que se dispone de forma transversal a la dirección de la lengua del glaciar rocoso con lo que coadyuva a su sujeción en la ladera; su presencia indicaría la sucesión clásica desde el modelado glaciar del entorno, al actual en el que predominan ya las condiciones de tipo periglaciar.

Entre el escarpe que es su área fuente y la raíz del glaciar rocoso se interpone un talud de derrubios. En el extremo oriental del escarpe que forma el área de alimentación del glaciar rocoso de Cerro Krund se localiza el término de uno de los remontes de la estación de esquí Complejo Cerro Castor. Esta circunstacia ha obligado al acondicionamiento de la pista de esquí y como resultado, la desaparición del talud de derrubios en ese sector: por ello, la raíz del glaciar rocoso está totalmente desconectada del talud lo cual implica un completo desabastecimiento de material, mediante crioclastia y caída por gravedad, por lo que sólo algún bloque de gran tamaño procedente de algún evento sísmico sería capaz de llegar hasta la raíz; además, el mantenimiento en uso de la pista 


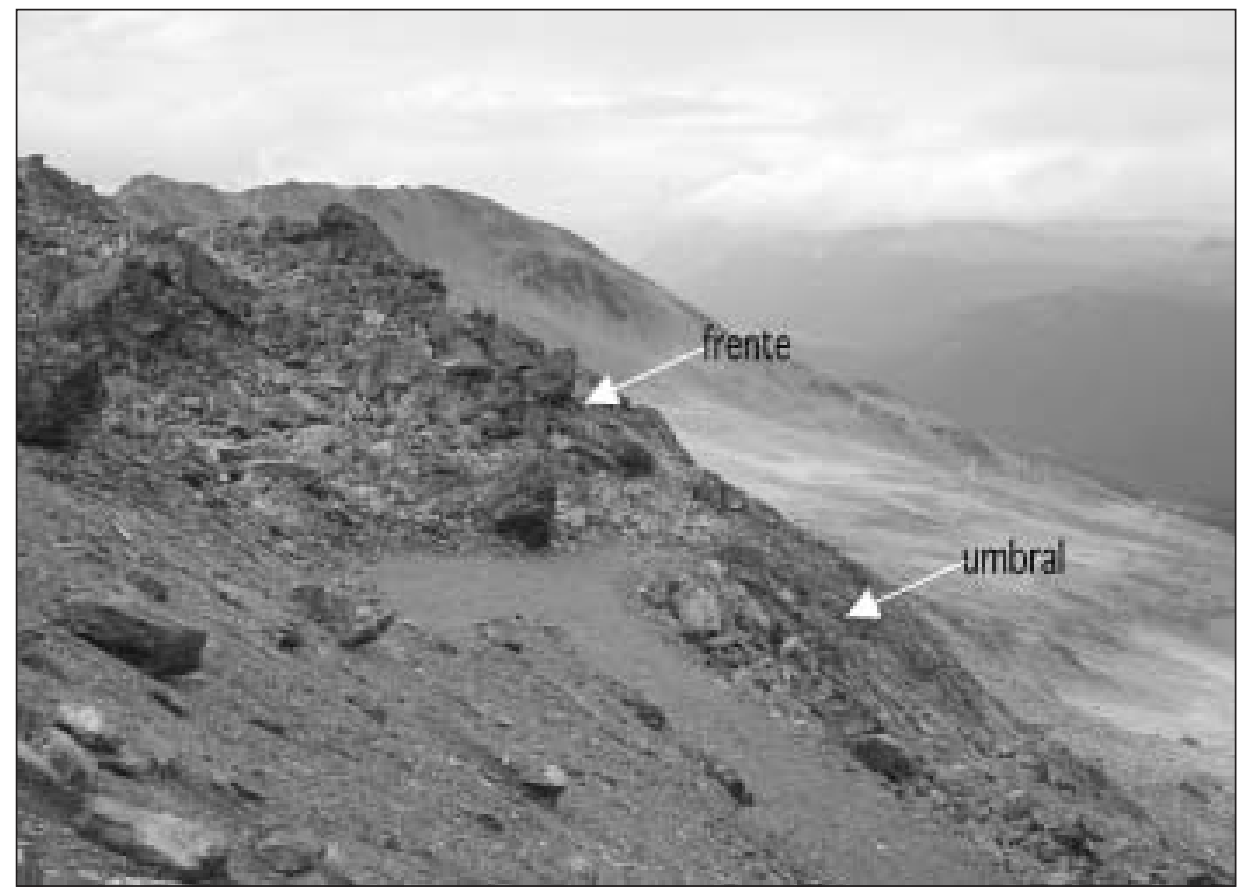

Foto 2. Vista desde el Oeste del frente del glaciar rocoso y del umbral de origen glaciar subyacente contra el que se ha desarrollado.

de esquí obliga a mantener limpio y despejado de bloques todo ese sector con lo cual el glaciar rocoso ha perdido una gran parte de su área fuente.

Los bloques situados en las crestas con una tonalidad más oscura aparecen colonizados por líquenes (además de líquenes, en torno a algunas fisuras y sectores de algunos bloques donde se retiene más humedad aparecen los musgos), lo cual indicaría una dinámica muy ralentizada del glaciar y unas condiciones subaéreas más prolongadas que hacen posible la colonización vegetal. Mientras que en los surcos los bloques de vulcanitas aparecen sin colonizar por los líquenes lo que se relaciona con una mayor persistencia nival en éstos.

En la pista de acceso al glaciar rocoso son visibles, tanto en el flanco Este como en el Oeste (Foto 3), una serie de grietas en cizalla de dirección transversal a la pista y que serían indicadores de que, con posterioridad a su construcción, el cuerpo central del glaciar rocoso aún experimenta movimiento a favor de la pendiente en dirección Norte-Sur. La presencia de esas grietas, junto con otros indicios como son la existencia de hielo intersticial, nos indica que se trata de un glaciar rocoso activo. En este sentido, el glaciar rocoso mantiene hielo intersticial a modo de matriz entre los bloques y entre la propia matriz fina que es visible desde la superficie a través de grietas; su existencia, aún a 


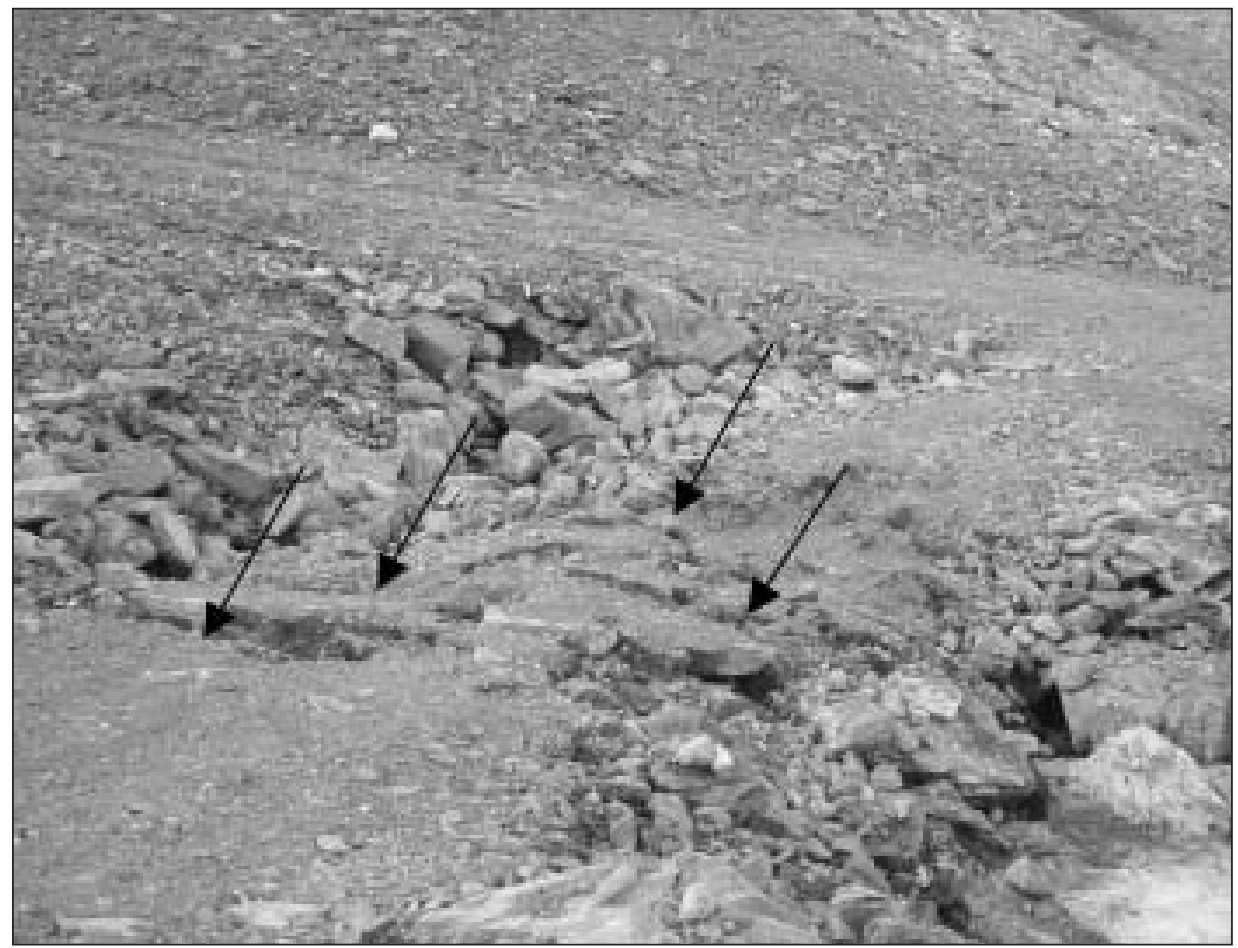

Foto 3. Detalle de las grietas en la pista de acceso (flechas negras) en el flanco Este del glaciar rocoso. Indican el reciente desplazamiento a favor de la pendiente del cuerpo central del glaciar.

finales de la estación cálida, implica unas condiciones morfoclimáticas de tipo periglaciar y que podamos catalogar el glaciar rocoso de Cerro Krund dentro de la categoría de los activos.

La observación detallada de la superficie del glaciar, sobre todo de sus surcos, transversales, permite apreciar alguna de sus características básicas: angulosidad como consecuencia del corto transporte, la nula colonización liquénica de los surcos y su heterometría, auque en superficie está ausente la fracción más fina que forma su matriz, como consecuencia de lavado continuo de las aguas de fusión nival. La aparente disposición caótica de los bloques que constituyen el glaciar esconde en ocasiones una disposición inclinada de los mismos con su dirección de buzamiento hacia la zona raíz, es decir hacia el Norte, el eje mayor paralelo o subparalelo a la del flujo que forma el surco y cresta del glaciar rocoso. También es perfectamente observable la disposición imbricada de los bloques y cantos (Foto 4), que indica la presencia de flujos compresivos responsables de la morfología de crestas y surcos que tiene el glaciar rocoso. 


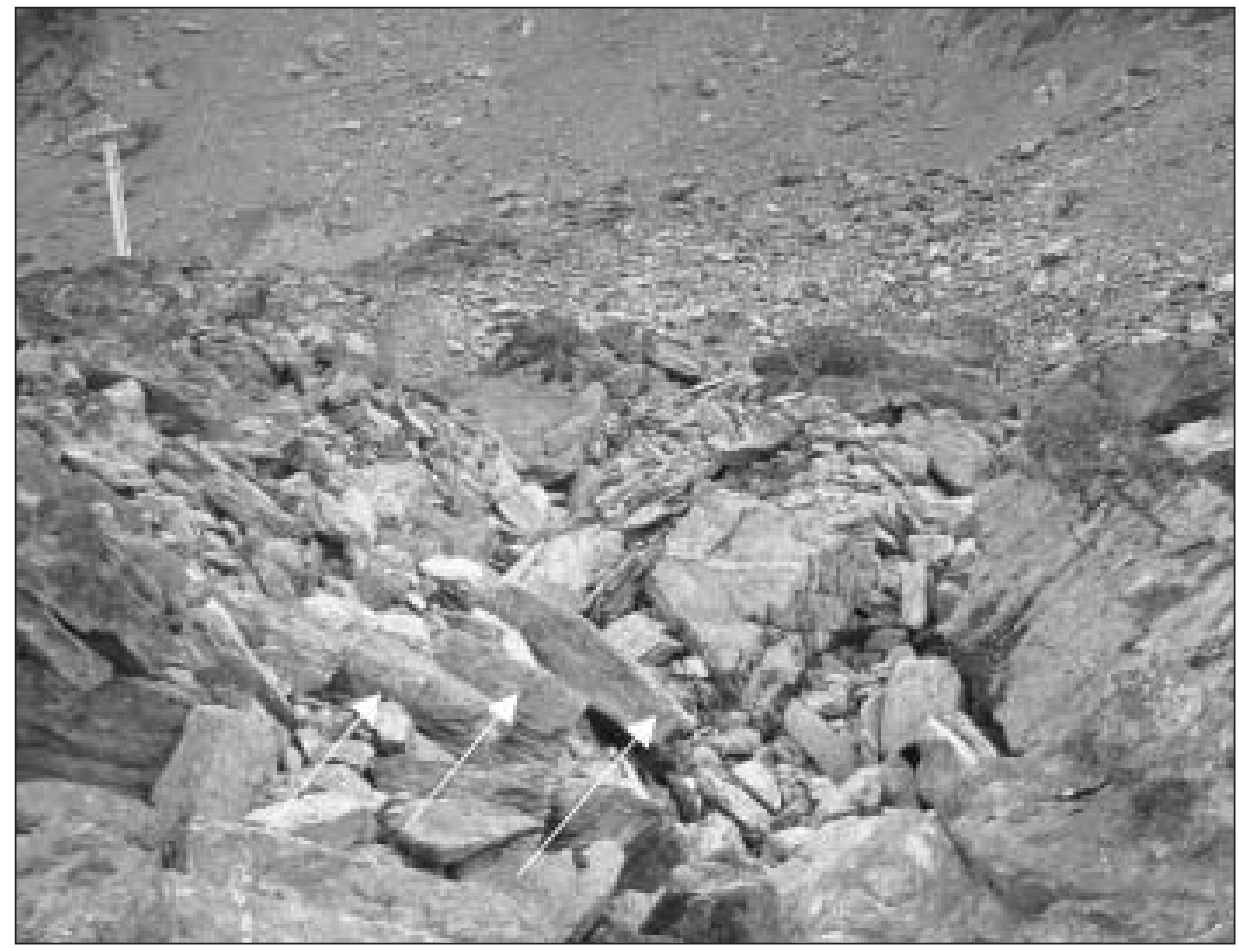

Foto 4. Detalle de la disposición de los bloques en uno de los surcos del glaciar rocoso (flechas blancas), mostrando su dirección de buzamiento hacia el Norte, zona raíz, y la imbricación de los mismos.

El glaciar rocoso de Cerro Krund se caracteriza por su elevada heterometría pues, junto a bloques de varias toneladas presenta una abundante matriz compuesta por fragmentos de pequeño tamaño incluso fracción fina. Las rocas volcanoclásticas y piroclásticas estratificadas que constituyen el basamento de la zona de Cerro Castor (Fm. Lemaire) arman fundamentalmente su área fuente. Se caracteriza, por un lado, por una alta e irregular densidad de fisuración, fruto de su también acusada tectonización y sometimiento a una elevada sismicidad y, por otro, por la fragmentación subsiguiente a la descompresión que ha experimentado el macizo como consecuencia del proceso de deglaciación reciente; ambas implican una elevada friabilidad y como consecuencia el suministro desde el talud de abundantes fragmentos muy heterogéneos. La matriz más fina se formaría como última etapa de la fragmentación física de esas vulcanitas.

\section{Discusión}

Desde el punto de vista dinámico, algunos autores hablan de active rock glacier y fossil según presente o no movimiento (Blagbrough, 1984), o de tres categorías (Barsch, 
1996), active, inactive y fossil o relict, para referirse, respectivamente, a los que presentan actividad y movimiento, a los que no se mueven pero contienen aún hielo en su interior y a los que no sólo no se mueven sino que no tienen hielo en su interior. La presencia de hielo intersticial aún a finales de la estación cálida y, sobre todo, la aparición de grietas en cizalla en la pista que lo atraviesa, y en ambos flancos del glaciar, indican el lento desplazamiento de su cuerpo central y que, por lo tanto, estamos ante un glaciar rocoso activo (Everdinguen, 1998).

En ese sentido la existencia en su superficie de surcos en forma de depresiones cerradas y crestas atenuadas de forma natural es el resultado de la presencia de flujo compresivo indicador de su actividad (Wahrhaftig \& Cox, 1959; Potter, 1972 y Haeberli, 1985).

En cuanto a la génesis del glaciar rocoso de Cerro Krund hay una serie de factores locales que convergen en su origen. En primer lugar, estamos en un ámbito montañoso que está sufriendo una rápida y reciente deglaciación, como lo prueba el marcado retroceso del pequeño glaciar de circo Alvear Este, situado en la misma alineación montañosa y cuyo frente se haya ya muy alejado y por encima de los cordones morrénicos atribuibles a la PEH. En las inmediaciones de Cerro Krund sólo persiste algún pequeño helero sobre las grietas de las zonas más escarpadas. La dinámica glaciar ha sido sustituida por la de tipo periglaciar y nival como manifiestan todo el cortejo de formas de detalle que aparecen en su entorno por encima del límite del bosque (suelos estriados y poligonales, bloques aradores, cantos y bloques gelifractados, pavimentos nivales). Por otro lado, la desaparición del glaciar de circo que ocupó ese espacio ha dejado un extenso escarpe en condiciones muy inestables por su verticalidad y por razones meramente lito-estructurales que ya comentamos anteriormente, a lo cual se une la elevada sismicidad regional. Se crean así unas condiciones óptimas, desaparecido el hielo glaciar, para que la vertiente sur del Cerro Krund se constituya como un área fuente de un glaciar rocoso con un elevado potencial morfogenético.

Algunos autores que han investigado los glaciares rocosos han destacado la influencia morfoestructural y la relación que existe entre las dimensiones del área de alimentación y las de los glaciares rocosos que se desarrollan a su pie, Wahrhaftig y Cox (1959), Barsch (1996), o bien la relación existente entre los glaciares rocosos y la presencia en su zona de alimentación de macizos muy fisurados por la tectónica, André, (1992), Evin (1985, 1987 y 1988).

La transformación de las condiciones estables en inestables como consecuencia de la fusión del hielo glaciar han servido para definir uno de los modelos (post glacial instability model, Burguer et alt., 1999), de la génesis de los glaciares rocosos. Las condiciones periglaciares que suceden a las de carácter glaciar no sólo suponen un cambio en el sistema morfogenético, sino que aquellas comienzan con un incremento de material disponible en el ámbito de los circos rocosos como consecuencia de la descompresión que experimentan las paredes de éstos ante la desaparición del hielo durante la deglaciación. Se trata de un enfoque que se basa en el comportamiento mecánico de las rocas (aunque el desencadenante sea la pérdida del hielo que comprime las estructuras y ello 
tenga que ver con cambios en las condiciones ambientales) que se ven sometidas a descompresiones locales y como consecuencia se ponen en marcha procesos y mecanismos que tienen a reequilibrar y a estabilizar los afloramientos rocosos.

Las vertientes rocosas ante la ausencia del hielo que las comprimía suelen ajustarse rápidamente a las nuevas condiciones por lo que tienden a alcanzar un estado de equilibrio en su inclinación; la naturaleza, escala y duración de este reajuste paraglaciar de las pendientes, está fuertemente condicionada por la estructura de la roca y, en especial, por la disposición, densidad y profundidad de la red de juntas o discontinuidades (Ballantyne, 2002).

Por otro lado, la superposición del glaciar rocoso de Cerro Krund a formas erosivas glaciares (su frente se apoya sobre un umbral transversal rocoso pulido por el hielo) y de acumulación (arcos morrénicos situados bajo ese mismo umbral), parecen indicar una génesis postglaciar bajo condiciones periglaciares.

En ese sentido, los recientes trabajos de campo emprendidos en la isla Disko, han constatado la existencia de dos tipos de glaciares rocosos (Humlum, 1996, 1999, 2000), los glacier-derived rock glacier, en los que aparecen núcleos de hielo que presentan una continuidad hasta el pequeño glaciar de cabecera (Humlum, 1996) y los talus-derived rock glacier de origen periglaciar. En todo caso, sin entrar en la controversia sobre su origen, parece que hilo conductor es el hecho de que los glaciares rocosos son básicamente el resultado de una localización bajo taludes con alto suministro de materiales en un ambiente de permafrost (Humlum, 2000).

La constatación de recientes movimientos en masa y avalanchas en esas laderas (Coronato y Roig, 1999, Moner, 2000), no empece el origen periglaciar del glaciar rocoso de Cerro Krund, al contrario. Su morfología en lengua (tongue shaped rock glaciers, Barsch, 1996) ha llevado a veces a su interpretación como movimientos en masa sobre las laderas, rockslides y landslides, con los que tienen cierto parecido (de hecho este uno de los orígenes de los glaciares rocosos para algunos autores como Johnson, 1987).

\section{Conclusiones}

Las evidencias geomorfológicas, tales como los surcos y crestas arqueadas, la inclinación hacia la zona raíz e imbricación de cantos y bloques, resultado del flujo compresivo, indican que estamos ante un glaciar rocoso. Por su forma en planta lo catalogamos como de lengua (tongue shaped rockglacier).

Su génesis implica unas condiciones morfoclimáticas actuales y subactuales de tipo periglaciar, al menos con permafrost discontinuo. Además, su situación al pié de un escarpe (Cerro Krund) en el seno de un circo previamente excavado por el hielo glaciar, es la característica de los glaciares rocosos. Su orientación al Sur, así como su situación topográfica y altitudinal, son factores que favorecen una escasa insolación y, por ello, la conservación del hielo intersticial que aún contiene. 
La elevada heterometría de los derrubios que lo forman, con abundante bloques de gran tamaño y varias toneladas de peso, indican que en el abastecimiento de material, además de fenómenos de alta frecuencia y baja intensidad (caídas por crioclastia desde el escarpe rocoso de Cerro Krund), han tenido que suceder otros de baja frecuencia y más alta intensidad, tales como movimientos en masa y desprendimientos inducidos por un lado por la descompresión general del macizo que sigue a la deglaciación y, sobre todo, por eventos sísmicos que son frecuentes en la región.

El acondicionamiento y mantenimiento de la pista de esquí afecta al abastecimiento de clastos desde el talud de derrubios en la parte más oriental de la raíz del glaciar rocoso. Ello implica una disminución en la entrada de material y, por tanto, la ralentización de su dinámica.

Creemos que sería interesante plantear el estudio a fondo del glaciar rocoso de Cerro Krund dentro del contexto regional por su significación climática, pues sospechamos que se sitúa en el límite de su "ámbito ecológico". En el mismo cordal montañoso hay algún glaciar rocoso (a $850 \mathrm{~m}$ en el entorno del Lago Alvear) que era bien visible en la foto aérea de 1988, pero que en la actualidad se encuentra en avanzado estado de desmantelamiento debido, probablemente, a la desaparición del sustrato helado, así como a la alta friabilidad del roquedo de esa zona.

En ese sentido, de confirmarse el rápido retroceso del frente del glaciar Alvear Este, el más cercano y el de mayor dimensión de la zona, significaría una elevación de la cota del permafrost discontinuo, por lo que el glaciar rocoso de Cerro Krund pasaría a corto plazo a una fase de inactividad y, posteriormente, al estado de glaciar rocoso fósil, alcanzado el cual, no está asegurada su conservación debido a la elevada fragmentación y friabilidad del material que lo compone.

\section{Agradecimientos}

Agradezco a los doctores A. Coronato y J. Rabassa, del CADIC, las atenciones recibidas durante el II Curso de Campo en Geomorfología y Geología del Cuaternario en Tierra del Fuego y a lo largo de mi estancia en Ushuaia durante febrero y marzo de 2004.

\section{Referencias bibliográficas}

ANDRE, M.F. (1992). Rock glaciers in central and north western Spitsbergen. Rev. Géom. Dynamique, 41 (2): 47-63.

Ballantyne, C.K. (2002). Paraglacial Geomorphology. Quaternary Science Revue, 21: 1935-2017

BARSCH, D. (1996). Rock glaciers. Indicators for the Present and Former Geoecology in High Mountain Environments. Springer, Berlin. 331 p. 
Blagbrough, J.W. (1984). Fossil rock glaciers on Carrizo Mountain, Lincoln County, New Mexico. New Mex. Geol., 6 : 65-68.

Burger, K.C., Degenhartd, J.J. y Giardino, J.R. (1999). Engineering geomorphology of rock glaciers. Geomorphology, 31: 93-132.

Coronato, A. (1995). The Last Pleistocene glaciation in tributary valleys of de Beagle Channel, Southternmost South America. Quaternary of South America and Antartic Peninsula 9. A.A. Balkema Plublishers, Rotterdam, p 173-182.

Coronato, A. (1996). Desarrollo de circos en el sector sudoccidental de los Andes Fueguinos. XIII Congreso Geológico Argentino, Actas IV, Buenos Aires, p: 347.

Coronato, A., y Roig, C. (1999). Peligro geomorfológico en ambientes de génesis reciente. Valles de Tierra Mayor y Río Olivia. I Congreso argentino de Geomorfología y Cuaternario, Sta. Rosa, La Pampa.

EVERDINGEN, R. Van (ed.), (1998). Multi-language glossary of permafrost and related ground-ice terms. Boulder, CO: National Snow and Ice Data Center/World Data Center for Glaciology.

Evin, M. (1985). Contribution à l'étude de la macrofissuration dans les parois situées à l'amont des glaciers rocheux des Alpes du Sud (France, Italie). Rèvue de Géomorphologie Dynamique, 34: 17-30.

Evin, M. (1987). Lithology and fracturing control of rock glaciers in southwestern Alps of France and Italy. In, J.R. Giardino, J.F. Shroder \& J.D. Vitek (eds.) Rock Glaciers. Allen \& Unwin, Boston, 83-106.

Evin, M. (1988). Repartition, morphologie et structure interne des glaciers rocheux des Alpes du Sud en fonction de la lithologie et de la fracturation. Bulletin du Centre de Géomorphologie du C.N.R.S., 34: 137-158.

HAEBerli, W. (1985). Creep of mountain permafrost: internal structure and flow alpine rock glaciers. Mitteilungen der Versuchsanstalt für Wasserbau, Hydrologie und Glaziologie, 77: 1-142

Humlum, O. (1996). Origin of Rock Glaciers: Observations from Mellemfjord, Disko Island, central West Greenland', Permafrost and Periglacial Processes, 7: 361-380.

Humlum, O. (1999). Late Holocene Climate in Central West Greenland: Meteorological Data and Rock Glacier Isotope Evidence. The Holocene 9 (5): 581-594.

Humlum, O. (2000). The Geomorphic Significance of Rock Glaciers: estimates of rock glacier debris volumes and headwall recession rates in $\mathrm{W}$ Greenland. Geomorphology, 35: 41-67. 
JoHnSON, P.G. (1987). Rock glacier: glacier debris systems or high-magnitude low- frequency flows?. In, J.R Giardino, J. F. Shroder Jr., \& J. D. Viteck (eds.), Rock Glaciers. Allen \& Unwin, London, 175-192.

Moner, I. (2000). Estudio de las avalanchas y los procesos de remoción en masa en el Complejo Invernal de Cerro Castor, Ushuaia, Argentina. (CADIC, Ushuaia). Unpublished report, $76 \mathrm{p}$.

PotTeR, N. (1972). Ice-cored rock glacier Galena Creek, Northern Absaroka Mountains, Wyoming. Geol. Soc. Am. Bull., 83: 3025-3058.

Rabassa, J., Coronato, A., Bujalesky, G., Saleme, M., Roig, C., Meglioli, A., Heusser, C., Gordillo, S., Roig, F., Borromei, A., y Quattrochio, M. (2000). Quaternary of Tierra del Fuego, Southernmost South America: an updated review. Quaternary International 68-71: 217-240.

Rabassa, J, Serrat, D., Heuser, C.J., Martí, C., y Coronato, A. (1990). The termination of the Pleistocene in the Beagle Channel, southernmost South America. In, Lundqvist, J. \& Saarnisto, M., (eds), IGCP projet 253:Termination of the Pleistocene. Geological Survey of Finland, Guide 31: 136-137.

Rabassa, J.O., (dir), (2004). Guía de Campo. II Curso de Campo en Geomorfología y Geología del Cuaternario de Tierra del Fuego. CADIC-CONICET, 147 p.

Tuhkanen, S. (1992). The climate of Tierra de Fuego from vegetation geographical point of view and its ecoclimatic counterparts elsewhere. Acta Bot. Fennica, 145: 164.

Wahrhafting, C. y Cox A. (1959). Rocks glaciers in Alaska Range. Geol. Soc. Am. Bull. 70: 383-436. 